Index Copernicus Value: 83.27

ISSN (e)-2347-176x ISSN (p) 2455-0450

crossref DOI: https://dx.doi.org/10.18535/jmscr/v5i3.36

\title{
A New Dermocosmetic Balm for the Management of Xerosis: Results from a Non-Interventional, Non-Comparative, Prospective Multicenter Study
}

Authors

\section{Floriane Gayraud ${ }^{1}$, Michèle Sayag ${ }^{2}$, Eric Jourdan ${ }^{3}$}

Naos, Laboratoire Bioderma, Lyon, 75, Cours Albert Thomas, 69447 Lyon Cedex 03, France

Corresponding Author

Floriane Gayraud

Naos, Laboratoire Bioderma, 75, Cours Albert Thomas, 69447 Lyon Cedex 03, France.

Email:e.jourdan@bioderma.com

\begin{abstract}
Introduction-Xerosis is a common skin condition affecting all skin types at all ages. However, itis somewhat more often observed in young children and the elderly.

Objective-To assess the safety and benefit of a dermocosmetic balm in the management of xerosis.

Methods-Non-interventional, non-comparative, prospective multicenter study conducted in xerosis subjects of at least 3 months of age. Clinical assessments included the investigator's evaluation of local tolerance, xerosis signs and symptoms at Days0, 14 and 21, as well as the subject's perception of improvement, satisfaction, and compliance.

Results- The mean age of the 1149 recruited subjects was $42.4 \pm 24.9$ years. According to the investigators, $882(76.8 \%)$ subjects had severe xerosis.

The local tolerance of SBT was excellent, with only $1 \%$ of the subjects reporting adverse events leading to withdrawal. All xerosis signs and symptoms had significantly $(p<0.0001)$ improved at all post-Day 0 visits, starting as early as Day 7.Xerosis had improved in 1127 (98.1\%) subjects and in 864 (75.2\%) subjects the disorder had completely disappeared.

Patient perception improved significantly (all p<0.0001) between Day 7 and Day 14/21 as well as between Day 14 and 21.In total, 1081 (94.1\%) subjects rated the efficacy of the dermocosmetic excellent or good, and 1032 (91.2\%) subjects were satisfied with the balm.

Conclusion-The local tolerance of the balm is excellent. The balm provides good clinical benefit in the management of xerosis.

Keywords- Balm, dry skin, SBT complex, skin barrier, xerosis.
\end{abstract}

\section{INTRODUCTION}

Xerosis, or dry skin, is a very common skin condition affecting all skin types at all ages. However, it is more frequently observed in young children and in the elderly in whom the skin is more fragile than in the active population. At birth, newborn skin is structurally and functionally immature compared to adult skin and has to undergo a physiological maturation process after birth. During this period, newborn skin is easily prone to xerosis. Conversely, in the elderly, xerosisis the result of age-related physiological changes of the skin (senile xerosis). ${ }^{1-3}$ 
Xerosis is caused by several physiopathological factors influencing the integrity of the skin barrier, resulting in modifications of the stratum corneum, the composition of intracellular lipids and of the hydrolipidic film, as well as the trans epidermal water loss and the cutaneous $\mathrm{pH} .{ }^{1,4}$ Depending on the pathology, there is a predominance of either keratinization or intercorneocytar lipid problems. Both types of abnormalities are found in all xerosis types. 1,5

It may be aninherited condition, such as ichthyosis, psoriasis or atopic dermatitis, or an acquired condition associated with different pathologies orphysiological disorders. These pathologies and disorders may be caused by endogenous (hormonal imbalances (menopause), pharmacological treatments (anti-cancer treatments, ${ }^{6,7}$ ) or exogenous factors, including meteorological conditions such as extreme temperature, wind, and excessive sun exposure. Moreover, it may be the result of personal hygiene habits, including the use of aggressive detergents, frequent washing or of other environmental factors including central heating and air conditioning., 1,9

In general, dry skin is dull and tight, and in very dry skin and in the elderly, it is often combined with pruritus. ${ }^{10,11}$ Chronic pruritus frequently leads to mechanical reactions such as scratching, rubbing or pinching. Scratching may induce variable types of damage to the skin including excoriations, crusts, lichenification, papules and nodules. When resolving, these lesions may result in hyper- or hypopigmentation and atrophic scars. Several lesions of different stages and sizes may co-exist in patients with chronic pruritus. ${ }^{12}$

Xerosisis not limitedto a single area of skin and may be observed on the upper and lower limbs as well as on the face, neck and on other sides of the body. Upon examination, either a thinning of the skinor, conversely, increased skin thickness is observed. In addition to visible signs, xerosis can also cause psychological stress and limit the individual's quality of life. ${ }^{1,13}$

In healthy skin, the predominance of hydrophobic substances (lipidic cement) in the intercellular constituents is a significant factor regulating the enzymatic activity and dehydration of the skin. Together with the intracellular constituents, the hydrophilic film is present on the skin as an emulsion and formed from sweat and sebum excretion. It covers the epidermis, thus strengthening the cutaneous barrier by helping the skin to defend the body against trans epidermal water loss or hyperhydration, particularly during climatic changes. ${ }^{1}$

The most common management of xerosis consists of using emollients and moisturizers. All of them improve the condition and provide temporary relief from signs and symptoms for patients by hydrating the skin, leading to swelling and a reduction of the surface undulations of the skin. ${ }^{14}$ As a result, xerosis improves rapidly in appearance, which is partly related to the smoothing effect of the products. ${ }^{15,16}$ However, when the application ofthese products is discontinued, a rapid relapse of xerosis is observed.

The investigated dermocosmetic moisturizing balm, hereafter "SBT complex" (Atoderm ${ }^{\circledR}$ Intensive balm, Naos, Laboratoire Bioderma, France), has been developed to manage durably dry skin. SBT complex contains Palmitoyl Ethanolamide (PEA) - an anti-pruritus agent, sucroesters, $\beta$-sitosterol-an anti-inflammatory agent - and zincgluconate, known for its antibacterial action, thereby allowing the management of the causes, signs and symptoms of the disorder. Unpublished data confirms that SBT complex biologically restores the natural skin barrier by inducing the synthesis of epidermal lipids and by improving the action on the skin barrier. SBT complex contains a multi-lamellar lipidic complex enhancing the integration of ceramides into the skin, as well as other active substances, thus restoring the deficient natural skin barrier.In a different formulation, SBT complex has demonstrated its benefit in the adjunctive management of atopic dermatitis. ${ }^{17}$

The objective of this non-interventional, prospective multicenter study was to assess the 
local tolerability and clinical benefit of a regular use of SBT complex balm in the management of different types of xerosis associated or not with pruritus.

\section{MATERIALS AND METHODS}

The study was conducted between November 2014 and June 2015 at more than 800 study sites in France according to Good Clinical Practices, legal local requirements for the conduct of observational studies and the principles of the Declaration of Helsinki.

Subjects of good general health and of at least 3 months of age had to present the day of inclusion with any type of xerosis, associated or not with pruritus.

Suitable subjects were asked to stop their current emollient, if any, and to apply the prescribed dermocosmetic on their skin at least once daily over 21 days.

At Day 0, the investigators collected demographic and other baseline data and conducted a general and xerosis-specific clinical evaluation in assessing the type of the xerosis, based on their experience as well as xerosis signsand symptoms (desquamation, roughness, fissures and scratching lesions, tightness, pruritus and insomnia) scored on a 5-point scale ranging from $0=$ none to $4=$ severe. The subject's efficacy perception of SBT complex balm and clinical signs and symptoms were rated on Days 7, 14 and 21. In addition, at Day 21, subjects reported the observed benefit on a scale ranging from "totally agree" to "do not agree at all" and assessed the improvement of their skin dryness, cosmetic properties of SBT complex balm and product compliance. They also reported possibly encountered side effects.

A descriptive statistical analysis was performed using $\mathrm{SAS}^{\circledR}$, version 9.4. A symmetrical test was used to compare results between Day7 and 14/21, and between Day 14 and 21. Variables were expressed as mean $\pm \mathrm{SD}$, medians, minimum and maximum values, $1^{\text {st }}$ and $3^{\text {rd }}$ quartiles and $95 \%$ confidence intervals. The risk was set at $5 \%$. Missing data was not replaced.

\section{RESULTS}

\section{Demography and baseline data}

In total, 1149 subjects were recruited for this study. Of those, 850 were females. The mean subject age was $42.4 \pm 24.9$ years.

According to the investigators, most subjects had severe xerosis $(882,76.8 \%)$; senile xerosis was reported for 141 subjects $(12.2 \%)$ and $17(1.5 \%)$ suffered from ichthyosis. A total of 109 (9.5\%) subjects with other types of xerosis were recruited. Overall, $481(42.5 \%)$ subjects had mild desquamation (mean score: 1.2 \pm 0.8$), 499$ (43.7\%) moderate roughness (mean score: 1.5 \pm 0.8 ), 456 $(40.6 \%)$ moderate sensation of tightness (mean score: $1.6 \pm 0.9)$. The majority of subjects (780; $69.3 \%$ ) had no fissures (mean score: $0.4 \pm 0.7$ ), 416 $(36.7 \%)$ had almost no lesions caused by itching (mean score: $1.1 \pm 0.9)$ while 438 (38.6\%) subjects reported moderate pruritus (mean score: $1.7 \pm 0.9$ ) and $822(73.4 \%)$ had no insomnia (mean score: $0.4 \pm 0.8$ ).Further demographic and baseline data including sign and symptom severity scores are detailed in table 1.

\section{Local Tolerance}

After 21 days, only 38 (3.3\%) subjects reported mild erythema with and without small papules and burning at mainly very dry application sites or on the face. Of those, 12 subjects, $1.0 \%$ of the overall study population, discontinued the SBT complex balm upon their request.

\section{Clinical Benefit}

Improvement of signs and symptoms was statistically significant $(\mathrm{p}<0.0001)$ at all postDay 0 visits starting as early as Day7 and sustaining until Day 21 for desquamation $(0.2 \pm 0.5)$, roughness $(0.4 \pm 0.6)$, lesions caused by scratching $(0.2 \pm 0.5)$, pruritus $(0.3 \pm 0.6)$, insomnia $(0.1 \pm 0.4)$, sensation of tightness $(0.2 \pm 0.5)$ and fissures $(0.1 \pm 0.3)$. Percent improvement after 7 , 14 and 21 days of application is given in Figure 1. 


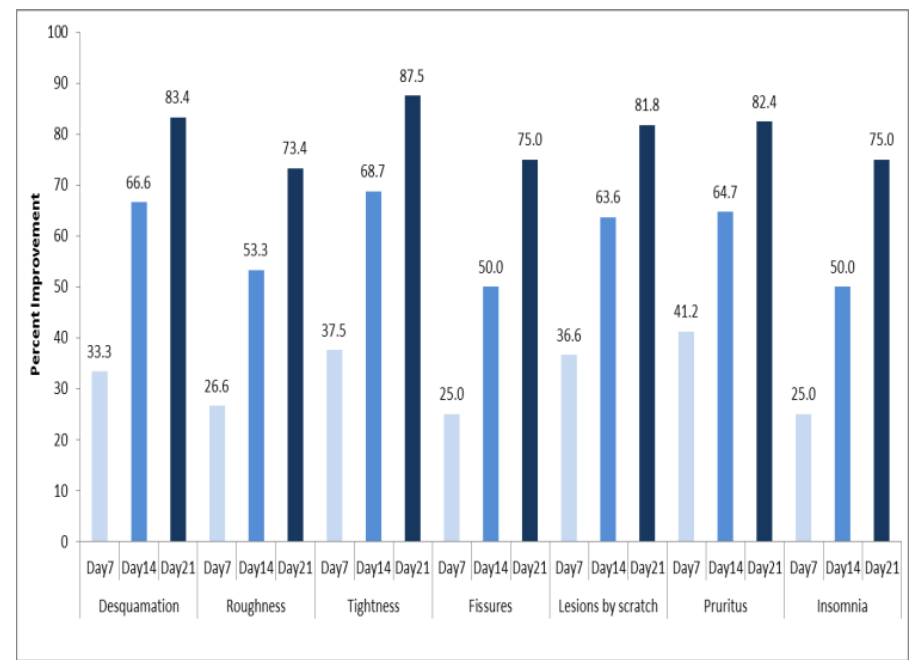

Figure 1 Percent improvement of signs and symptoms at Day 7, 14 and 21 from Day 0

Improvement for all signs and symptoms was statistically significant $(\mathrm{p}<0.0001)$ as early as day 7.

Overall, xerosis had improved between Day 7 and Day 14/21, as well as between Day 14 and Day 21 according to the subjects; all differences were statistically significant compared to Day7 (all $\mathrm{p}<0.0001)$. Details are provided in Figure 2.

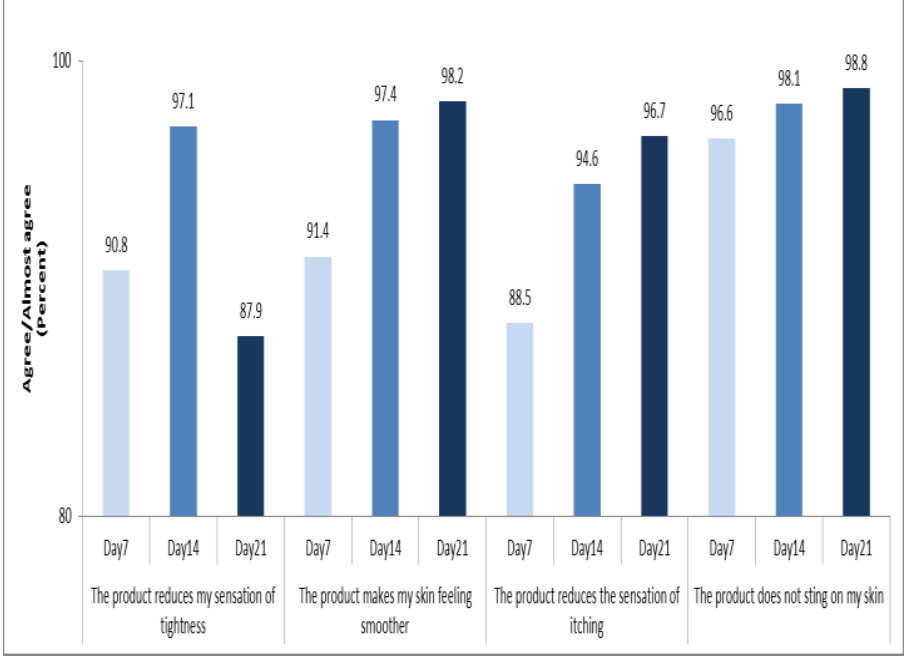

Figure 2 Subject efficacy perception in percent (selected data for agree/almost agree)

Differences between Day 14/21 and Day 7 as well as between Day 14 and Day 21 were statistically significant (all $\mathrm{p}<0.0001)$

According to $1127(98.1 \%)$ subjects, SBT complex balm had at least improved theirxerosisincluding $864(75.2 \%)$ subjects in whom the disorder had completely disappeared. In total, $94.1 \%$ (1081) of the subjects rated the efficacy of SBT complex balm excellent or good.

A large majority of subjects $(836,72.8 \%)$ applied SBT complex balm once daily, 250 (21.8\%) subjects applied the balm twice daily; the remaining subjects $(62,5.4 \%)$ applied the product at least three times daily.

After 21 days of daily use, 1032 (91.2\%) subjects were satisfied with SBT complex balm.

Table 1-Demographic and Baseline Data

\begin{tabular}{|c|c|}
\hline Parameter & Value \\
\hline Gender (n,\%) & $\mathrm{N}=1129$ \\
\hline Male & $294(26 \%)$ \\
\hline Female & $835(74 \%)$ \\
\hline Age (years) & $\mathrm{N}=1149$ \\
\hline Mean \pm SD & $42.4 \pm 24.9$ \\
\hline Median & 44.9 \\
\hline Min/Max & $0.3 / 99.7$ \\
\hline CI $[95 \%]$ & $41.0 / 43.9$ \\
\hline Type of dry skin & $\mathrm{N}=1149$ \\
\hline Severe dry skin with pruritus & $882(76.8 \%)$ \\
\hline (idiopathic cause) & $141(12.2 \%)$ \\
\hline Senile xerosis & $17(1.5 \%)$ \\
\hline Ichtyosis & $109(9.5 \%)$ \\
\hline Multiple origins of dry skin & \\
\hline Desquamation* & \\
\hline Mean \pm SD & $1.2 \pm 0.8$ \\
\hline Median & 1.0 \\
\hline Min/Max & $0.0 / 3.0$ \\
\hline CI [95\%] & $1.1 / 1.2$ \\
\hline Roughness* & \\
\hline Mean \pm SD & $1.5 \pm 0.8$ \\
\hline Median & 2.0 \\
\hline Min/Max & $0.0 / 3.0$ \\
\hline CI [95\%] & $1.5 / 1.5$ \\
\hline Sensation of tightness* & \\
\hline Mean \pm SD & $1.6 \pm 0.9$ \\
\hline Median & 2.0 \\
\hline Min/Max & $0.0 / 3.0$ \\
\hline CI [95\%] & $1.5 / 1.6$ \\
\hline Fissures* & \\
\hline Mean \pm SD & $0.4 \pm 0.7$ \\
\hline Median & 0.0 \\
\hline Min/Max & $0.0 / 3.0$ \\
\hline CI $[95 \%]$ & $0.4 / 0.5$ \\
\hline Lesions caused by scratching* & \\
\hline Mean \pm SD & $1.1 \pm 0.9$ \\
\hline Median & 1.0 \\
\hline Min/Max & $0.0 / 3.0$ \\
\hline CI [95\%] & $1.0 / 1.1$ \\
\hline Pruritus* & \\
\hline Mean \pm SD & $1.7 \pm 0.9$ \\
\hline Median & 2.0 \\
\hline Min/Max & $0.0 / 3.0$ \\
\hline CI [95\%] & $1.7 / 1.8$ \\
\hline Insomnia* & \\
\hline Mean \pm SD & $0.4 \pm 0.8$ \\
\hline Median & 0.0 \\
\hline Min/Max & $0.0 / 3.0$ \\
\hline CI $[95 \%]$ & $0.4 / 0.4$ \\
\hline
\end{tabular}

$*$ Assessed on a scale from $0=$ none to $4=$ severe 


\section{DISCUSSION AND CONCLUSION}

The present prospective observational study assessed the beneficial effect of a novel dermocosmetic balm (Atoderm ${ }^{\circledR}$ Intensive Balm, Naos, Laboratoire Bioderma, France) in the management of xerosis. The investigated balm contains palmitoylethanolamide (PEA) an anti-pruritus agent, sucroesters, $\beta$-sitosterol an antiinflammatory agent and zincgluconate known for its antibacterial action.

More than 800 investigators recruited 1149 subjects with xerosis. At Baseline, xerosis was rated by the investigators as severe. Thisclinical assessment of the xerosis type at Day 0 contrasts with assessments of signs and symptoms made by using a 5-point scale showing that the majority of subjects only suffered from mild to moderate xerosis and may therefore be considered with caution. Indeed, the notable difference in severity of the condition evaluated by the investigators based on their experience and that of based on an objective assessment using a scale of the individual xerosis signs and symptoms confirm the importance of objective measurements in the daily clinical practice: objective measurements allow for selecting the most adapted skin care regimen.

Regardless of this issue, results after 21 days of a mainly one-daily-application of the balm confirmed the excellent local tolerability of the balm. The balm reduced significantly all xerosis signs and symptoms in almost all patients; more than $75 \%$ of the subjects considered that their disorder had completely disappeared confirming the efficacy of SBT complex balm.

Truly, xerosis is not a life-threatening disorder. However, when evolving into more severe stages, it may cause pruritus, lesions and fissures which, when remained unmanaged, may lead to disruption of the natural skin barrier, inflammation and potentially to infection by pathogens. Hence, acute care relieving from signs and symptoms are desirable, but, restoring the natural skin barrier function without inducing local tolerability issues is undeniably more important.
To date, a whole armada of emollients, hydrants and moisturizers are available for managing the signs of xerosis. Though, only a certain category of cosmetic products, called dermocosmetics, also allow for a persistent improvement of the condition and a significant relief from signs and symptoms. In addition to their smoothing and hydrating ingredients, dermocosmetics contain active substances allowing managing effectively and safely xerosis which support the efficacy of pharmacological treatments. ${ }^{17}$

The present results confirm that the regular use of a dermocosmetic balm developed for the management of xerosisis safe, improves signs and symptoms and may initiate the restoration of the natural skin barrier, even though we agree that the non-comparative design of the study gives rise for limitations and that a long-term follow-up investigation should confirm this allegation.

High subject satisfaction is an important issue conditioning compliance. The fact that more than $90 \%$ of the subjects were highly satisfied and regularly applied the balm may have played a role in the very positive study outcome and may support an increased clinical benefit in the daily care of the condition.

In conclusion, the present study demonstrated that according to the subjects and to the investigators, a once-daily application during 21 days of SBT complex balm is safe and reduces significantly signs and symptoms of xerosis while providing a large clinical benefit resulting in a complete clearance of the condition in more than $75 \%$ of the subjects. A large majority of subjects appreciated the product allowing for a regular use of the balm and hence supporting an increased clinical benefit.

\section{ACKNOWLEDGEMENTS}

The study was granted by Naos, Laboratoire Bioderma, France. All authors are employees of Naos, Laboratoire Bioderma, Lyon, France.

The authors acknowledge the writing assistance of Patrick Göritz, SMWS, France. 


\section{REFERENCES}

1. Pons-Guiraud A. Dry skin in dermatology: a complex physiopathology. J Eur Acad Dermatol Venereol. 2007;21 Suppl 2:1-4. DOI: 10.1111/j.1468-3083.2007.02379.x

2. Al-Nuaimi Y, Sherratt MJ, Griffiths CE. Skin health in older age. Maturitas. 2014;79(3):256-64.

DOI: 10.1016/j.maturitas.2014.08.005

3. Blume-Peytavi U, Tan J, Tennstedt D, Boralevi F, Fabbrocini G, Torrelo A, et al. Fragility of epidermis in newborns, children and adolescents. J Eur Acad Dermatol Venereol. 2016;30 Suppl 4:356.doi: $10.1111 / j d v .13636$.

4. Saint-Leger D, Francois AM, Leveque JL, Stoudemayer TJ, Kligman AM, Grove G. Stratum corneum lipids in skin xerosis. Dermatologica. 1989;178(3):151-5.

5. White-Chu EF, Reddy M. Dry skin in the elderly: complexities of a common problem. Clin Dermatol. 2011;29(1):3742. DOI: $10.1016 /$ j.clindermatol. 2010.07.005

6. Al-Ghazzewi FH, Tester RF. Impact of prebiotics and probiotics on skin health. Benef Microbes. 2014;5(2):99-107. DOI: 10.3920/BM2013.0040

7. Zouboulis CC, Boschnakow A. Chronological ageing and photoageing of the human sebaceous gland. Clin Exp Dermatol. 2001;26(7):600-7.

8. Hoffman L, Subramanyan K, Johnson AW, Tharp MD. Benefits of an emollient body wash for patients with chronic winter dry skin. Dermatol Ther. 2008;21(5):41621 DOI: $\quad 10.1111 / \mathrm{j} .1529-$ 8019.2008.00225. $\mathrm{x}$

9. Wolf R, Parish LC. Effect of soaps and detergents on epidermal barrier function. Clin Dermatol. 2012;30(3):297-300. DOI: 10.1016/j.clindermatol.2011.08.021

10. Ward JR, Bernhard JD. Willan's itch and other causes of pruritus in the elderly. Int $\mathrm{J}$
Dermatol. 2005;44(4):267-73. DOI: 10.1111/j.1365-4632.2004.02553.x

11. Valdes-Rodriguez R, Stull C, Yosipovitch G. Chronic pruritus in the elderly: pathophysiology, diagnosis and management. Drugs Aging. 2015;32(3):201-15. DOI: $10.1007 / \mathrm{s} 40266-015-0246-0$

12. Stander S, Weisshaar E, Mettang T, Szepietowski JC, Carstens E, Ikoma A, et al. Clinical classification of itch: a position paper of the International Forum for the Study of Itch. Acta Derm Venereol. 2007;87(4):291-4.

DOI: 10.2340/00015555-0305

13. Humbert P, Dreno B, Krutmann J, Luger TA, Triller R, Meaume S, et al. Recommendations for managing cutaneous disorders associated with advancing age. Clin Interv Aging. 2016;11:141-8. DOI: 10.2147/CIA.S96232

14. Marks R. Actions and effects of emollients. New York. Georg Thieme Verlag S, 2002. 12-23.

15. Loden M. The clinical benefit of moisturizers. J Eur Acad Dermatol Venereol. 2005;19(6):672-88; quiz 86-7. DOI: $10.1111 /$ j.1468-3083.2005.01326.x

16. Lodén M. Role of Topical Emollients and Moisturizers in the Treatment of Dry Skin Barrier Disorders. American Journal of Clinical Dermatology. 2003;4(11):771-88.

17. Gayraud F, Sayag M, Jourdan E. Efficacy and tolerance assessment of a new type of dermocosmetic in infants and children with moderate atopic dermatitis. J Cosmet Dermatol. 2015;14(2):107-12. DOI: 10.1111/jocd.12145. 